\title{
Uso em Longo Prazo de Anticoagulantes Orais na Cardiologia
}

\author{
Prolonged Use of Oral Anticoagulants in Cardiology \\ Renata Martins Almeida ${ }^{1}$, Gilson Soares Feitosa ${ }^{{ }^{*}}$ \\ Serviço de Cardiologia do Hospital Santa Izabel; Salvador, Bahia, Brasil
}

Correspondence addresses:

Dr. Gilson Soares Feitosa

gilson-feitosa@uol.com.br

Received: December 10, 2019

Revised: January 13, 2020

Accepted: January 15, 2020

Published: March 31, 2020

Data Availability Statement: All relevant data are within the paper and its Supporting Information files.

Funding: This work was the result of authors' initiative. There was no support of research or publication funds.

Competing interests: The authors have declared that no competing interests exist.

Copyright

(C) 2020 by Santa Casa de Misericórdia da Bahia.

All rights reserved.

ISSN: 2526-5563
Os anticoagulantes orais são medicações amplamente utilizadas na cardiologia. Durante muito tempo, a varfarina foi a única opção disponível no mercado para esta terapia, tendo ainda hoje papel singular em determinadas situações. Porém, com o surgimento dos anticoagulantes orais de ação direta (DOACs), no século $\mathrm{XXI}$, o tratamento anticoagulante vem apresentando grande avanço, dispondo de novas opções terapêuticas que ofertam mais segurança e efetividade à terapia. Diversos cenários da cardiologia requerem o seu uso e, recentemente, muitos estudos têm sido realizados a fim de esclarecer os riscos e benefícios de sua empregabilidade, a segurança e eficácia dos fármacos disponíveis em variadas situações clínicas, bem como a interação dessas drogas com outras medicações amplamente utilizadas na cardiologia. Dessa forma, os DOACs vêm cada vez mais ganhando espaço nos diversos cenários, apresentando maior segurança, menor perfil de interações medicamentosas e alimentares, maior comodidade posológica, acarretando maior adesão dos pacientes à terapia, além de melhor eficácia, se comparados à varfarina. É importante ressaltar que o uso dessa nova classe de droga está reservado a situações específicas, tendo em vista que não mostrou benefício em determinadas situações e, em outras situações, essas drogas precisam ser estudadas para terem seu uso validado na prática clínica. O objetivo dessa revisão é promover uma atualização, à luz dos principais e mais robustos ensaios clínicos publicados até o momento, sobre o uso dos anticoagulantes orais nos diversos cenários da cardiologia, abordando as suas principais indicações, contraindicações, assim como o tempo de uso dessa terapia e orientações posológicas em cada situação, com base nas melhores e mais atuais evidências sobre o tema.

Palavras-chave: Anticoagulação em Cardiologia; Cumarínicos; Anticoagulantes de Ação Direta; Novos Anticoagulantes; Fibrilação Atrial.

Oral anticoagulants are medications widely used in cardiology. For a long time, warfarin was the only option available on the market for this therapy, and today it still has a unique role in some cases. However, with the emergence of direct oral anticoagulants (DOACs) in the $21^{\text {st }}$ century, anticoagulant treatment has had a big breakthrough, constituting a new therapeutic option that offers more safety and effectiveness for anticoagulant therapy. Several cardiology scenarios require its uses. Recently, many studies have been carried out in order to clarify the risks and benefits of their use, the safety and efficiency of the drugs available in many clinical situations, and the interaction of these drugs with other medications commonly used in cardiology. Thus, DOACs are increasingly finding spaces in several settings, presenting safety, a lower profile of drug and food interactions, a better dosage convenience, adhesion of patients to the therapy, and efficiency when compared to 
warfarin. The use of this new class of drugs is reserved for specific situations, considering that it didn't show any benefits in some conditions, and in many situations, these drugs need to be studied to have their use validated in clinical practice. The purpose of this review is to promote an update of the use of oral anticoagulants in many cardiology scenarios, considering the most robust clinical trials published to date to discuss the main indications, contraindications, as well as, time of use and dosage guidelines in each situation.

Keywords: Anticoagulation in Cardiology; Oral Anticoagulants; Direct Oral Anticoagulants; Warfarin, Atrial Fibrillation.

\section{Introdução}

Os anticoagulantes orais são ferramentas terapêuticas fundamentais na cardiologia. $\mathrm{O}$ uso desta terapia é de grande importância em determinadas situações, como na prevenção dos fenômenos tromboembólicos relacionados a condiçõesclínicascomoafibrilaçãoatrial,próteses valvares, e também as associadas a cardiopatias estruturais ou secundárias a complicações de um infarto, entre outras situações clínicas.

Por mais de 50 anos, os antagonistas da vitamina $\mathrm{K}$ foram os únicos anticoagulantes orais disponíveis. Mais de um milhão de prescrições de varfarina são dispensadas anualmente nos Estados Unidos, ficando essa substância entre as 15 drogas mais prescritas, em termos de quantidade. ${ }^{1}$ Em 1999, a varfarina foi o $11^{\circ}$ fármaco mais prescrito nos EUA. ${ }^{2}$ Ainda nos anos 90, o estudo MESA, mostrou que a terapia de anticoagulação teve um grande impacto, reduzindo em até 3 vezes a mortalidade da FA. ${ }^{3}$ Porém, no século XXI, com o advento de novos anticoagulantes orais, atualmente denominados anticoagulantes orais diretos, ou DOACs, o tratamento anticoagulante vem sendo revolucionado, sendo demonstrado em estudos clínicos: segurança, eficácia e superioridade com relação ao uso da varfarina. Assim, os DOACs estão ganhando cada vez mais espaço no cenário da cardiologia.

Os antagonistas da vitamina $\mathrm{K}$ apresentam algumas limitações que acabam dificultando seu uso na prática clínica, como por exemplo: a resposta ao medicamento é imprevisível, possuem uma janela terapêutica estreita (na maior parte dos casos o RNI alvo é entre 2-3), possuem início de efeito e neutralização do mesmo após retirada, lentos, apresentam interação com uma grande variedade de alimentos e outras drogas, e, por fim, temos que lidar ainda com a questão da resistência à varfarina e à necessidade de monitorização constante com ajuste frequente das doses. ${ }^{4}$

$\mathrm{O}$ controle do RNI representa um grande desafio em relação ao uso da varfarina. Se fizermos um comparativo do TTR (tempo na faixa terapêutica), ou seja, o tempo em que o RNI permanece entre 2-3 em paciente em uso de varfarina, portadores de fibrilação atrial, essa taxa nos estudos clínicos é algo em torno de $66 \%$, enquanto que na prática clínica essa taxa cai para $44 \%$ apenas. ${ }^{5,6}$

Em 2010, realizou-se uma análise de subgrupo de TTR médio por país em ensaios clínicos e observou-se que o Brasil apresenta uma taxa de apenas 54\% e já a Suécia apresenta uma taxa de $77 \%$. Isso é reflexo das condições socioeconômicas, do nível de educação da população e das políticas públicas em saúde. ${ }^{7}$

A literatura demonstra que em seguimento em longo prazo, a taxa de adesão a medicamentos diversos é um problema médico para todos os produtos, reduzindo ainda mais no decorrer do tempo, sendo um problema ainda pior no caso da varfarina. ${ }^{8}$

Os anticoagulantes orais diretos vêm revolucionando o tratamento anticoagulante, demonstrando uma ação mais previsível, melhor eficácia e maior segurança em comparação com a varfarina, por dispensar o monitoramento contínuo e apresentar uma menor interação medicamentosa e alimentar (Figura 1).

Os DOACs agem de maneira específica na cascatadecoagulação. Deuma formasimplificada, o produto final da cascata é a ativação do fator II 
(trombina), mediado pela ativação do Fator X. A varfarina age tanto na via intrínseca quanto na extrínseca, inibindo a ativação dos fatores II, VII, IX e X de maneira menos específica. Já os anticoagulantes de ação direta, por sua vez, agem diretamente na inibição do fator II (dabigatrana) e do Fator Xa (os demais). ${ }^{9-11}$

Se compararmos as duas estratégias, os DOACs possuem um início de ação muito mais rápido que a varfarina, além de uma meia vida mais curta. A reversão da varfarina com plasma fresco congelado ou vitamina $\mathrm{K}$ era considerada uma vantagem da varfarina, superada a partir de 2015 com o surgimento de anticorpos monoclonais específicos para a dabigatrana (idarucizumabe) e da rivaroxaban (andexanet alfa - ainda não disponível no Brasil). ${ }^{11-13}$

Os cenários da utilização crônica dos anticoagulantes orais na cardiologia podem ser assim descritos:

1.Anticoagulação oral na fibrilação atrial.

1.1. Situações especiais em anticoagulação oral na fibrilação atrial.

1.1.1. Anticoagulação na fibrilação atrial e Intervenção coronariana percutânea ou síndrome coronariana aguda.

1.1.2. Anticoagulação em pacientes com fibrilação atrial e doença arterial coronária crônica.

2. Anticoagulação oral em portadores de valvulopatias.

2.1. Anticoagulação oral em portadores de prótese valvar mecânica.

2.2. Anticoagulação oral em portadores de prótese valvar biológica.

2.3. Anticoagulação oral em pacientes submetidos a reparo valvar.

2.4. Anticoagulação oral em portadores de estenose mitral em ritmo sinusal.

3. Anticoagulação oral em portadores de síndrome coronariana aguda (SCA) sem outras indicações de anticoagulação.

4. Anticoagulação oral em portadores de insuficiência cardíaca em ritmo sinusal.
5. Anticoagulação oral em portadores de cardiopatia chagásica crônica em ritmo sinusal.

6. Anticoagulação oral em portadores de trombo em ventrículo esquerdo.

7. Anticoagulação oral em portadores de miocardiopatia não compactada.

8. Anticoagulação oral em portadores de cardiomiopatia hipertrófica.

9. Anticoagulação oral em pacientes submetidos a implante transcatéter de válvula aórtica (TAVI).

\section{Anticoagulação Oral na Fibrilação Atrial}

As primeiras evidências de que a terapia anticoagulante em pacientes com fibrilação atrial (FA) traz benefícios, ou seja, reduz a incidência de acidente vascular cerebral (AVC), vem de estudos antigos que compararam a varfarina, único anticoagulante oral existente na época, versus placebo nesse grupo de pacientes. Nesse cenário, a varfarina mostrou uma grande eficácia. ${ }^{14}$

Entretanto, esse tratamento pode ter consequências graves, como eventos hemorrágicos. Por este motivo, foi notada a importância de classificar os pacientes quanto ao risco de acidente vascular cerebral (AVC) e embolia sistêmica e identificar aqueles para os quais a terapia anticoagulante está indicada para prevenção desses eventos, assim como evitar o uso desse tratamento em pacientes de baixo risco.

Com a finalidade de predizer o risco cardioembólico, foram elaborados, no decorrer do tempo, alguns escores de risco. Nesse contexto, surgiu o escore $\mathrm{CHADS}_{2}$, publicado em 2001, que tem fácil aplicação na prática clínica e mostrou-se ser uma boa ferramenta para a predição do risco de AVC em pacientes com FA de origem não-reumática. ${ }^{15}$ Posteriormente, esse escore foi refinado para o $\mathrm{CHA}_{2} \mathrm{DS}_{2} \mathrm{VASc}$, tendo em vista o fato que o $\mathrm{CHADS}_{2}$ classifica uma grande parte os pacientes com FA como 
Figura 1. Mecanismo de ação dos anticoagulantes.

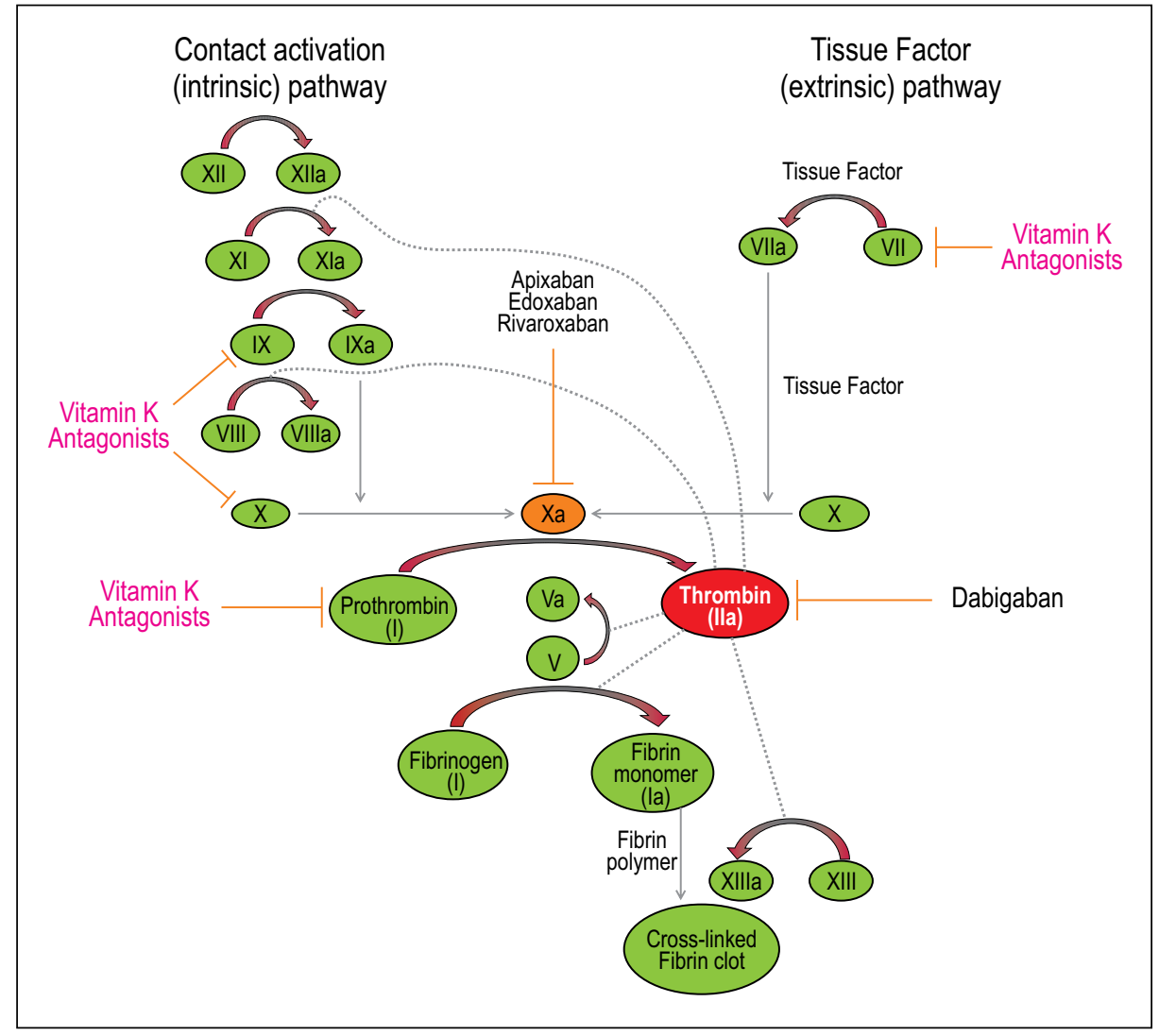

risco moderado e uma parte destes poderia ser, na verdade, de baixo risco, sendo exposto aos riscos da terapia anticoagulante sem comprovado benefício clínico. ${ }^{16,17}$

Através de fatores de risco sabidamente envolvidos com FA, o paciente é pontuado entre 0 e 6 (CHADS) e entre 0 e 9 ( $\left.\mathrm{CHA}_{2} \mathrm{DS}_{2} \mathrm{VASc}\right)$. Quanto maior o valor numérico, maior a taxa de eventos cardioembólicos em um ano em pacientes com FA não-valvar. ${ }^{17,18}$ Atualmente, devido a maior acurácia, utiliza-se rotineiramente o escore $\mathrm{CHA}_{2} \mathrm{DS}_{2} \mathrm{VASc}$, que reestratificou os pacientes de risco moderado no CHADS (pontuação de 1). ${ }^{16}$

Nos ensaios clínicos, determinou-se por definição a FA como valvar e não-valvar. Como valvar foi definida a presença de prótese mecânica e estenose mitral significativa. FA nãovalvar os demais casos, mesmo com outras lesões valvares presentes. A anticoagulação deve ser considerada para pacientes de risco moderado, ou seja, com $\mathrm{CHA}_{2} \mathrm{DS}_{2} \mathrm{VASc}$ de 1 ponto. Porém, pacientes do sexo feminino só são consideradas de risco moderado se $\mathrm{CHA}_{2} \mathrm{DS}_{2} \mathrm{VASc}$ for igual a 2 pontos. Já aqueles com alto risco, ou seja, com $\mathrm{CHA}_{2} \mathrm{DS}_{2} \mathrm{VASc} \geq 2$ pontos, a terapia anticoagulante está indicada devido ao benefício clínico comprovado na prevenção de eventos tromboembólicos. O sexo feminino só é considerado de alto risco se $\mathrm{CHA}_{2} \mathrm{DS}_{2} \mathrm{VASc} \geq 3$ pontos. $^{18}$

Para auxiliar os médicos na predição de eventos hemorrágicos em pacientes em uso de anticoagulantes, alguns escores estão disponíveis. O escore HAS-BLED, publicado em 2010, após a identificação de características associadas a um risco elevado de sangramento (hipertensão, função renal ou hepática alteradas, AVC prévio, sangramento prévio, razão normalizada internacional (RNI) lábil, idade $>65$ anos e uso de outras medicações ou álcool). Um escore $\geq 3$ pontos define o paciente como alto risco de 
sangramento. Atualmente, é o mais utilizado na prática clínica. $\mathrm{O}$ escore HASBLED discrimina pacientes com maior risco de fenômenos hemorrágicos. É importante ressaltar que uma pontuação elevada no escore HASBLED não está relacionada à recomendação de não utilização do anticoagulante em pacientes de risco elevado de fenômenos tromboembólicos. Para pacientes de maior risco de eventos hemorrágicos, estratégias de redução de risco de sangramento e acompanhamento mais rigoroso devem ser adotadas. ${ }^{19}$

No século XXI, surgiram os DOACs como alternativa a varfarina, em pacientes com FA não valvar (exclui portadores de próteses valvares e estenose mitral moderada a grave) com risco de moderado a alto de eventos embólicos. Ensaios clínicos foram realizados para avaliar sua eficácia e segurança nesses pacientes.

O estudo RE-LY, publicado em 2009, mostrou que a dabigatrana na dose de $110 \mathrm{mg} 2 \mathrm{x} /$ dia ou $150 \mathrm{mg} 2 \mathrm{x} /$ dia é não inferior à varfarina para prevenção de AVC ou embolia sistêmica. A dose mais alta da dabigatrana foi associada a um menor risco de acidente vascular cerebral ou embolia sistêmica. Já a dose mais baixa da dabigatrana foi associada a uma menor ocorrência de eventos hemorrágicos. É importante observar que a população que participou desse estudo era de menor risco, possuindo $\mathrm{CHADS}_{2} 0$ ou $1 .^{20}$

O estudo Rocket AF, publicado em 2011, mostrou que em pacientes com $F A$, a rivaroxabana na dose de $20 \mathrm{mg} /$ dia foi não inferior à varfarina para prevenção de AVC ou embolia sistêmica, mostrando também um melhor perfil de segurança dessa droga. ${ }^{21}$

O estudo Aristotle, publicado também em 2011, mostrou que a apixabana na dose de $5 \mathrm{mg}$ duas vezes ao dia, foi superior à varfarina na prevenção de AVC ou embolização sistêmica, com um melhor perfil de segurança. A dose foi reduzida para $2,5 \mathrm{mg}$ duas vezes ao dia se o paciente possuir duas das seguintes características: quando em pacientes $<60 \mathrm{~kg}$, creatinina $>1.5 \mathrm{ou}$ idade $>80$ anos. $^{22}$
Por fim, o estudo Engage AF - TIMI 48, publicado em 2013, mostrou que a edoxabana na dose de $60 \mathrm{mg} /$ dia foi não inferior à varfarina na prevenção de eventos embólicos. Além disso, ambos os regimes, 30 ou $60 \mathrm{mg} / \mathrm{dia}$, diminuíram significativamente as taxas de sangramentos. ${ }^{23}$

No conjunto da evidência, analisando a população dos diversos trabalhos, os DOACs têm um melhor perfil de segurança e eficácia que a varfarina. Entretanto, não existem trabalhos comparando os DOACs entre si. A apixabana, em pacientes de maior risco de sangramento, parece oferecer maior segurança. A escolha da terapia deve envolver a experiência do médico e as particularidades do paciente. ${ }^{24}$

Situações Especiais em Anticoagulação Oral na Fibrilação Atrial

\section{Anticoagulação na Fibrilação Atrial e Intervenção Coronariana Percutânea ou Síndrome Coronariana Aguda}

Pacientes portadores de FA, que requerem anticoagulação oral crônica, podem necessitar de intervenção coronariana percutânea (ICP) na vigência ou não de uma síndrome coronariana aguda, com necessidade de tripla terapia (anticoagulante oral + dupla antiagregação plaquetária). Essa situação eleva exponencialmente o risco de complicações hemorrágicas.

As recomendações das atuais diretrizes internacionais, com destaque para a última diretriz de FA da Sociedade Europeia de Cardiologia(ESC), publicada em 2017, sustentam o uso de terapia tripla com dupla antiagregação plaquetária + anticoagulante oral por algum período, variando de 1 a 6 meses, a depender do risco de sangramento do paciente. ${ }^{25,26}$

Estudos realizados recentemente têm nos mostrado que a terapia dupla iniciada precocemente com um DOAC somado ao inibidor P2Y12 está associada a menor ocorrência de sangramentos maiores, sem elevar 
a ocorrência de eventos isquêmicos, levando a crer que possivelmente irá ocorrer uma mudança nas futuras diretrizes. ${ }^{27-30}$

Dentre esses estudos, o estudo Pioneer AF PCI, publicado em 2016, comparou as estratégias de anticoagulação oral (antagonista da vitamina $\mathrm{K}$ em tripla terapia com aspirina mais inibidor $\mathrm{P} 2 \mathrm{Y} 12$ versus rivaroxabana em dupla terapia com uma dose de $15 \mathrm{mg} /$ dia mais inibidor P2Y12 ou em tripla terapia na dose de $2,5 \mathrm{mg} 2 \mathrm{x} /$ dia mais inibidor P2Y12 mais aspirina) em pacientes com FA submetidos a ICP com colocação de stent. O desfecho primário foi de segurança. $\mathrm{O}$ estudo mostrou que os esquemas com rivaroxabana foram associados com menor taxa de sangramento clinicamente significativo do que a terapia padrão com a varfarina, apresentando ainda uma eficácia semelhante nos três grupos. Porém essa dose de $2,5 \mathrm{mg}$ não está disponível comercialmente. ${ }^{27}$

O estudo Re-dual PCI, publicado em 2017, comparou dois regimes de dupla terapia antitrombótica que inclui dabigatrana nas doses de $110 \mathrm{mg} 2 \mathrm{x} / \mathrm{dia}$ ou $150 \mathrm{mg} 2 \mathrm{x} / \mathrm{dia}+$ um inibidor P2Y12 com o uso de tripla terapia antitrombótica com varfarina mais inibidor P2Y12 mais aspirina, em pacientes com FA submetidos a ICP. O desfecho primário foi de segurança. ${ }^{28}$

$\mathrm{O}$ estudo mostrou que a dupla terapia com dabigatrana e inibidor do $\mathrm{P} 2 \mathrm{Y} 12$ pós ICP apresentou um melhor perfil de segurança, reduzindo eventos hemorrágicos, sem alterar também a eficácia da terapia de anticoagulação. Porém, houve uma maior tendência a ocorrência de eventos isquêmicos com a dose de dabigatrana de $110 \mathrm{mg} .{ }^{28}$

O estudo Entrust AF PCI, publicado em 2019, comparou a edoxabana $60 \mathrm{mg} /$ dia em combinação com o inibidor P2Y12 com a tripla terapia envolvendo a varfarina mais inibidor P2Y12 mais aspirina, em pacientes com FA submetidos a ICP. O estudo mostrou que nessa população, a edoxabana foi não inferior ao antagonista de vitamina $\mathrm{K}$, em relação à taxa de sangramento. Entretanto, houve uma tendência maior de eventos hemorrágicos no grupo de pacientes que fizeram uso da varfarina. Esse foi o único estudo que não mostrou superioridade da terapia envolvendo um DOAC nesse contexto. ${ }^{29}$

$\mathrm{O}$ estudo AUGUSTUS, publicado em março de 2019, é o maior e o melhor desenhado trabalho que avalia o uso dos DOACs nesse contexto. Esse estudo comparou apixabana $5 \mathrm{mg}$ 2x/dia mais inibidor P2Y12 com um antagonista da vitamina $\mathrm{K}$ mais inibidor P2Y12 e também a eficácia e segurança de baixa dose de aspirina em comparação com placebo em pacientes com FA e síndrome coronariana aguda (SCA) recente ou ICP. Foram recrutados 4.614 pacientes, randomizados em média após uma semana do evento índice. O desfecho primário foi de segurança. ${ }^{30}$

$\mathrm{O}$ estudo mostrou que os pacientes que usaram apixabana sangraram menos do que os pacientes que usaram varfarina. $\mathrm{E}$ os pacientes que usaram a tripla terapia (anticoagulante mais inibidor P2Y12 e aspirina) tiveram mais eventos hemorrágicos do que os pacientes que fizeram uso da dupla terapia, usando o placebo ao invés da aspirina. Em relação aos desfechos de eficácia, a ocorrência de morte ou hospitalização foi maior no grupo de pacientes que fez uso do antagonista da vitamina $\mathrm{K}$ e, também, no grupo que fez tripla terapia com o uso da aspirina, sem diferenças significativas na incidência de eventos isquêmicos. ${ }^{30}$

Dessa forma, esse estudo foi um grande marco para a cardiologia, podendo ser a base para mudanças futuras nas diretrizes mundiais sobre o tema.

Anticoagulação em Pacientes com Fibrilação Atrial e Doença Arterial Coronariana Crônica

O estudo AFIRE, publicado em setembro de 2019, avaliou a monoterapia com rivaroxabana versus a terapia dupla com rivaroxabana mais aspirina ou inibidor da $\mathrm{P} 2 \mathrm{Y} 12 \mathrm{em}$ pacientes com fibrilação atrial e doença arterial coronariana (DAC) mais de um ano após a revascularização ou naqueles com confirmação angiográfica de DAC 
sem necessidade de revascularização. $O$ desfecho primário foi de eficácia e de segurança. ${ }^{31}$

$\mathrm{O}$ estudo mostrou que a incidência de eventos isquêmicos foi semelhante em ambos os grupos, mostrando que a rivaroxabana é não-inferior à terapia combinada para eficácia. Já em relação à segurança, ocorreu maior quantidade de eventos hemorrágicos com o uso da terapia combinada, mostrando que a monoterapia com a rivaroxabana é superior para o desfecho de segurança. ${ }^{31}$

Dessa forma, o estudo AFIRE confirma o que já vem sendo feito na prática clínica. Em pacientes portadores FA e doença arterial coronariana crônica em tratamento clínico ou tratados invasivamente (ICP ou cirurgia de revascularização miocárdica) há mais de 1 ano, é razoável o uso de monoterapia com anticoagulante oral, sem necessidade do uso de terapia antiplaquetária.

\section{Anticoagulação Oral em Portadores de Valvulopatias}

\section{Anticoagulação Oral em Portadores de Prótese} Valvar Mecânica

Outro cenário bastante frequente e de extrema importância na cardiologia é a presença de prótese valvar mecânica. Embora as próteses valvares mecânicas ofereçam maior durabilidade, expõem o paciente a elevado risco de tromboembolismo. $\mathrm{Na}$ posição mitral, o risco de formação de trombos é duas vezes maior em relação à posição aórtica. ${ }^{32}$

Atualmente, as principais diretrizes sobre este tema recomendam anticoagulação com antagonistas da vitamina $\mathrm{K}$ (AVK) e monitorização do RNI em pacientes com prótese valvar mecânica. ${ }^{33}$

Uma meta de RNI entre 2-3 é recomendada para prótese valvar aórtica mecânica e nenhum fator de risco para tromboembolismo. ${ }^{33}$

Uma meta de RNI entre 2,5-3 é recomendada para portadores de prótese valvar aórtica mecânica e fatores de risco adicionais para eventos tromboembólicos (FA, tromboembolismo prévio, disfunção de ventrículo esquerdo ou condições hipercoaguláveis) ou pacientes com prótese valvar mecânica de geração mais antiga (como bola-gaiola). Além disso, a meta de RNI entre 2,5 e 3,5 também é recomendada para a prótese valvar mecânica mitral. ${ }^{33}$

Uma observação pertinente a ser feita é que as diretrizes recomendam aspirina de $75 \mathrm{mg}$ a $100 \mathrm{mg}$ por dia, além da anticoagulação com um antagonista da vitamina $\mathrm{K}$ (AVK) em pacientes com prótese valvar mecânica. ${ }^{33}$

É importante ressaltar que a terapia de anticoagulação oral com inibidores diretos da trombina não deve ser usada em pacientes com próteses valvares mecânicas. ${ }^{33}$

O estudo RE-ALIGN, publicado em 2013, comparou a dabigatrana (em 3 esquemas diferentes - $150 \mathrm{mg} / 220 \mathrm{mg} / 300 \mathrm{mg}$ ) à varfarina em pacientes com valva protética mecânica. $\mathrm{O}$ estudo foi interrompido precocemente devido às taxas inaceitáveis de eventos tromboembólicos (AVC, infarto agudo do miocárdio e trombose de prótese) e hemorrágicos no grupo dabigatrana. ${ }^{34}$

Estudos com inibidores do fator Xa estão em curso.

Anticoagulação Oral em Portadores de Prótese Valvar Biológica

Em relação aos portadores de prótese valvar biológica, sabe-se que as biopróteses são menos trombogênicas que as próteses mecânicas. Ocorre ainda que há uma maior incidência de eventos tromboembólicos nos 6 primeiros meses após o implante da prótese. ${ }^{35}$

É importante destacar que pacientes com fibrilação atrial e biopróteses valvares devem usar anticoagulantes orais independentemente do escore $\mathrm{CHA}_{2} \mathrm{DS}_{2}$-VASc. ${ }^{33}$

As principais diretrizes sobre o tema orientam que anticoagulação com AVK para obter um RNI de 2,5 (2,0-3,0) é razoável por pelo menos 3 meses e estendendo-se por até 6 meses após a cirurgia de troca valvar mitral ou aórtica por prótese biológica em pacientes com baixo risco de sangramento. ${ }^{33,36}$ 
Outra orientação dessas diretrizes é que a aspirina $75 \mathrm{mg}$ a $100 \mathrm{mg}$ por dia é razoável em todos os pacientes com prótese biológica aórtica ou mitral. ${ }^{33}$

Atualmente, as Diretrizes não recomendam o uso de DOACs em pacientes portadores de válvulas bioprotéticas. ${ }^{33,36}$

Uma análise desse subgrupo de pacientes do estudo Engage, ou seja, envolvendo pacientes portadores de bioprótese valvar e fibrilação atrial, foi publicada em 2017. Destaca-se que no estudo original só foram incluídos pacientes com mais de 30 dias após cirurgia de troca valvar. $\mathrm{O}$ desfecho primário foi de eficácia e segurança. ${ }^{37}$

O grupo tratado com edoxabana apresentou taxas similares de AVC/eventos embólicos sistêmicos e sangramento maior em comparação com a varfarina, sendo que o grupo tratado com uma dose menor (30mg/dia) de edoxabana apresentou taxa um pouco mais baixa de sangramento maior. ${ }^{37}$

O MACE (infarto agudo do miocárdio, AVC ou morte cardiovascular) e o resultado clínico líquido primário foram menores no grupo da edoxabana. ${ }^{37}$

Outra análise desse subgrupo de pacientes foi realizada no estudo ARISTOTLE, sendo comparada a apixabana com a varfarina em pacientes portadores de bioprótese valvar e FA. O desfecho primário foi de eficácia (AVC/embolia sistêmica) e segurança (sangramentos graves). ${ }^{38}$

Astaxas geraisdeeventos clínicosforambaixas, sem diferença significante entre a apixabana e a varfarina para quaisquer resultados. ${ }^{38}$

Esses resultados sugerem então que os DOACs parecem ser uma alternativa razoável à varfarina em pacientes com FA e bioprótese valvar. Em 2016, foi realizado o primeiro estudo mundial desenhado com a finalidade de comparar o uso da varfarina com a dabigatrana em portadores de fibrilação atrial e bioprótese valvar cardíaca, o estudo DAWA. ${ }^{39}$ Ao realizar esta comparação, os desfechos de trombose valvar ou intracardíaca, embolia para o sistema nervoso central ou periférica, após 3 meses de seguimento, foram similares entre os grupos. No entanto, este foi um estudo pequeno. Dessa forma, são necessários ensaios clínicos randomizados maiores para avaliar a segurança e eficácia dos DOACs nessa população de alto risco. ${ }^{39}$

Atualmente, encontra-se em andamento o estudo River (ensaio clínico randomizado, fase 2, aberto, de não inferioridade), para explorar a segurança e eficácia de rivaroxabana versus AVK em portadores de FA e bioprótese.

Alguns especialistas já estão fazendo uso dos DOACs como conduta off-label como uma alternativa à varfarina nesse grupo de pacientes, tendo apresentado bons resultados.

É válido chamar a atenção para o fato de que no cenário de pós-operatório imediato de cirurgia de troca valvar por prótese biológica, em que é recomendado o uso de terapia anticoagulante por 3-6 meses, a droga recomendada e segura é a varfarina, pois os estudos supracitados excluem esse grupo de pacientes.

\section{Anticoagulação Oral em Pacientes Submetidos} a Reparo Valvar

Em pós-operatório de cirurgia de reparo valvar, as principais diretrizes recomendam para pacientes submetidos a reparo cirúrgico da válvula mitral anticoagulação com um AVK (RNI 2,0-3,0) nos primeiros três meses, seguido de aspirina 50 a $100 \mathrm{mg}$ por dia iniciada após os primeiros três meses. Pacientes submetidos a reparo cirúrgico da válvula aórtica prescinde-se da anticoagulação e utiliza-se a aspirina de 50 a $100 \mathrm{mg}$ por dia. ${ }^{33,36}$

Anticoagulação Oral em Portadores de Estenose Mitral em Ritmo Sinusal

As principais diretrizes recomendam anticoagulação com AVK (RNI 2,0 -3,0) em pacientes com estenose mitral (EM) reumática moderada a grave e uma ou mais das seguintes condições: evento embólico anterior, trombo em átrio esquerdo ou diâmetro atrial esquerdo $>55 \mathrm{~mm}^{33,36,40}$ 
$O$ uso de anticoagulação em pacientes com EM em ritmo sinusal com aumento do átrio esquerdo ou contraste ecocardiográfico espontâneo no ecocardiograma transesofágico é controverso, uma vez que a eficácia dessa terapêutica nessa situação é incerta. Essa recomendação só é encontrada na diretriz do Chest. ${ }^{40}$

\section{Anticoagulação Oral em Portadores de Síndrome Coronariana Aguda (SCA) Sem Outras Indicações de Anticoagulação}

Após uma síndrome coronariana aguda, os pacientes permanecem em risco de recorrência de eventos cardiovasculares apesar do emprego da terapia de dupla-antiagregação plaquetária em longo prazo. ${ }^{41}$

Porém, a anticoagulação oral, em pacientes que não possuem outra indicação, após fase aguda de um evento coronariano agudo, não é recomendada em diretrizes atualmente. ${ }^{36}$

O estudo Appraise-2, publicado em 2011, comparou apixabana versus placebo, em adição a mono ou dupla terapia antiplaquetária, para prevenir morte cardiovascular, infarto agudo do miocárdio não fatal ou $\mathrm{AVC}$ em portadores de síndrome coronariana aguda. $\mathrm{O}$ desfecho primário foi de eficácia e segurança. Porém, o estudo foi interrompido precocemente pois aumentou as taxas de sangramento em pacientes de alto risco pós síndrome coronariana aguda, sem reduzir eventos isquêmicos. ${ }^{42}$

Outro estudo, o ATLAS ACS 2 -TIMI 51, publicado em 2012, comparou a rivaroxabana (em duas esquemas não habituais diferentes $-2,5 \mathrm{mg}$ e $5 \mathrm{mg}$ duas vezes ao dia) versus placebo, em adição a dupla terapia antiplaquetária, em pacientes com síndrome coronariana aguda recente.

O desfecho primário foi de eficácia e segurança. ${ }^{43}$ Ambas as dosagens reduziram o desfecho primário de mortalidade cardiovascular, infarto agudo do miocárdio ou AVC e trombose de stent. ${ }^{43}$
A baixa dose de rivaroxabana reduziu mortalidade cardiovascular e mortalidade por todas as causas. Porém, a rivaroxabana aumentou taxa de sangramento não fatal, incluindo hemorragia intracraniana, mas não houve aumento na taxa de sangramento fatal. ${ }^{43}$

No entanto, em 2012, a FDA rejeitou o uso de rivaroxabana em pacientes com SCA devido a preocupações com dados faltantes: $15 \%$ dos pacientes descontinuaram prematuramente o estudo, sendo que $8,3 \%$ retiraram o consentimento informado e houve uma alta taxa de seguimento incompleto e taxas de morte não contabilizadas. ${ }^{43}$

\section{Anticoagulação Oral em Portadores de Insuficiência Cardíaca em Ritmo Sinusal}

Outro cenário da cardiologia são os pacientes portadores de insuficiência cardíaca (IC). Devido ao baixo débito cardíaco, a diminuição da atividade física, ao edema periférico e a formação de trombos em câmaras cardíacas, esses pacientes apresentam altas taxas de fenômenos tromboembólicos. ${ }^{44}$

Existem dados escassos sobre o uso dos DOACs em pacientes com IC em ritmo sinusal, porém, na prática clínica, essa conduta off-label já está sendo tomada em situações selecionadas. O estudo WASH, publicado em 2004, comparou aspirina versus varfarina versus placebo em pacientes portadores de insuficiência cardíaca e disfunção ventricular esquerda que requerem diureticoterapia.

O estudo não mostrou diferenças significativas entre os grupos de tratamento na frequência do desfecho primário composto de morte, infarto do miocárdio não fatal ou acidente vascular cerebral não fatal. A hospitalização por razões cardiovasculares (especialmente piora da IC) foi mais frequente em pacientes randomizados para aspirina..$^{45}$

O estudo WATCH, publicado em 2009, comparou varfarina (RNI 2,5-3,0) com aspirina $162 \mathrm{mg} / \mathrm{dia}$ ou oclopidogrel $75 \mathrm{mg} / \mathrm{dia}$ em 
pacientes com insuficiência cardíaca sintomática há pelo menos 3 meses, em ritmo sinusal e fração de ejeção de ventrículo esquerdo menor ou igual a $35 \%$.

O desfecho primário foi de eficácia e não houve diferenças significativas entre os três grupos de tratamento para o desfecho primário composto por primeira ocorrência de morte, infarto do miocárdio não fatal ou acidente vascular cerebral não fatal. ${ }^{46,47}$

O estudo WARCEF, publicado em 2012, comparou a varfarina (RNI alvo 2,5 -3) com aspirina $(325 \mathrm{mg} / \mathrm{dia})$ em pacientes portadores de IC em ritmo sinusal e fração de ejeção do ventrículo esquerdo $<35 \%$.

O desfecho primário foi de eficácia (AVC, hemorragia intracraniana ou morte por qualquer causa). ${ }^{48}$ Não ocorreu diferença significativa no desfecho primário entre a varfarina e a aspirina. Um risco reduzido de AVC com a varfarina foi alcançado por um risco aumentado de sangramento grave. ${ }^{47}$

As diretrizes recomendam que em pacientes portadores de insuficiência cardíaca em ritmo sinusal, a anticoagulação deve ser reservada para pacientes com tromboembolismo prévio ou trombose mural. ${ }^{36,48}$

Atualmente, portanto, a anticoagulação plena é indicada para pacientes com IC em ritmo sinusal, somente como profilaxia secundária para eventos tromboembólicos. ${ }^{36}$

\section{Anticoagulação Oral em Portadores de Cardiopatia Chagásica Crônica em Ritmo Sinusal}

Outro cenário da cardiologia é a cardiopatia chagásica. Pacientes portadores dessa entidade apresentam com frequência discinesias e aneurismas ventriculares, dilatação das câmaras cardíacas, estase venosa, fibrilação atrial, elevando dessa forma, a incidência de fenômenos embólicos. Porém, essa condição é bastante negligenciada no cenário mundial, existindo poucos estudos sobre o tema. ${ }^{49}$
Um estudo brasileiro, desenvolvido pela FIOCRUZ, publicado em 2008, mostrou o desenvolvimento de um escore para avaliar o risco de AVC em pacientes portadores de cardiopatia chagásica. Esse escore envolve pacientes que apresentam disfunção ventricular ( 2 pontos), aneurisma apical (1 ponto),alteração primária da repolarização ventricular ( 1 ponto) e idade $>48$ anos (1 ponto). Pacientes possuem indicação de terapia de anticoagulação se o escore for maior ou igual a 3 pontos. Essa recomendação consta na Diretriz Brasileira, com grau de evidência IIb. $^{50}$

Obviamente, a diretriz brasileira recomenda a terapia anticoagulante com AVK em pacientes portadores de cardiopatia chagásica crônica em ritmo sinusal que apresentem trombose mural ou tenham apresentado um evento embólico prévio. ${ }^{36}$

Atualmente, não é recomendado o uso dos DOACs em pacientes portadores de cardiopatia chagásica, pois não existem estudos envolvendo o uso dessas medicações nesse grupo de pacientes. ${ }^{36}$

\section{Anticoagulação Oral em Portadores de Trombo em Ventrículo Esquerdo}

Outra situação frequente é a presença de trombo em ventrículo esquerdo. Pacientes que possuem trombo no ventrículo esquerdo no contexto de uma síndrome coronariana aguda, devem receber anticoagulação com varfarina (RNI alvo: 2-3) por pelo menos 3-6 meses. Já pacientes portadores de IC, doença de chagas ou outras miocardiopatias devem receber anticoagulação crônica com varfarina (RNI alvo: 2-3), segundo as recomendações da Diretriz Brasileira deAntiagregantes Plaquetários e Anticoagulantes em Cardiologia. ${ }^{36}$

Não estão disponíveis ainda grandes estudos comparando a varfarina com DOACs nesse contexto. Assim, recomenda-se o uso da varfarina nessas condições. 


\section{Anticoagulação Oral em Portadores de Miocardiopatia Não Compactada}

Outro cenário é a miocardiopatia não compactada, que é uma anormalidade rara, de etiologia ainda desconhecida.

A prevenção das complicações tromboembólicas nessa entidade têm sido alvo de intensos debates. Alguns autores recomendam anticoagulação profilática, em longo prazo, para todos os pacientes com o diagnóstico de não compactação ventricular, independentemente da função ventricular. ${ }^{51}$

Porém, a recomendação atual da diretriz brasileira de IC para terapia de anticoagulação em pacientes portadores dessa patologia é: fibrilação atrial (independente do $\mathrm{CHA}_{2} \mathrm{DS}_{2}$-VASc), fração de ejeção $<40 \%$, tromboembolismo prévio ou trombo mural. ${ }^{51,52}$

\section{Anticoagulação Oral em Portadores de Cardiomiopatia Hipertrófica}

A fibrilação atrial na cardiomiopatia hipertrófica revela-se de alto risco para o surgimento de AVC.

Em pacientes portadores de cardiomiopatia hipertrófica, é recomendada anticoagulação oral crônica em pacientes que apresentem qualquer episódio de fibrilação atrial, mesmo que isolado, com varfarina (RNI alvo 2-3), independente do $\mathrm{CHA}_{2} \mathrm{DS}_{2}$-VASc. ${ }^{53,54}$

$\mathrm{Na}$ prática clínica, os DOACs estão sendo usados nessa população com bons resultados, porém, ainda não existem estudos comprovando a eficácia e segurança desses medicamentos nessa população.

\section{Anticoagulação Oral em Pacientes Submetidos a Implante Transcatéter de Válvula Aórtica (TAVI)}

Em relação aos pacientes submetidos a TAVI, é recomendado o uso de dupla anti-agregação plaquetária (clopidogrel $75 \mathrm{mg} /$ dia + aspirina
$100 \mathrm{mg} / \mathrm{dia}$ ) apenas nos primeiros 6 meses. 33,36 $\mathrm{O}$ estudo GALILEO, publicado em novembro de 2019 , comparou a rivaroxabana $10 \mathrm{mg} / \mathrm{dia}$ + aspirina $100 \mathrm{mg} / \mathrm{dia} \mathrm{x}$ aspirina $100 \mathrm{mg} / \mathrm{dia}+$ clopidogrel $75 \mathrm{mg} /$ dia, em pacientes submetidos a TAVI, sem indicação de anticoagulação por outra causa. Os desfechos primários foram de eficácia e segurança. ${ }^{55}$

$\mathrm{O}$ estudo foi encerrado precocemente pois a estratégia com rivaroxabana foi associada a um aumento nos principais eventos cardiovasculares adversos, aumento na mortalidade por todas as causas e aumento nos principais eventos hemorrágicos. ${ }^{55}$

\section{Referências}

1. Feldstein AC, Smith DH, Perrin N, Yang X, Simon SR, et al. Reducing warfarin medication interactions: an interrupted time series evaluation. Arch Intern Med. 2006;166(9):1009-15.

2. Shapiro SS. Treating thrombosis in the $21^{\text {st }}$ century. N Engl J Med 2003;349:1762-1764.

3. Vidaillet H, Granada JF, Chyou Po, Maassen K, Ortiz M, Pulido JN, et al.A population-based study of mortality among patients with atrial fibrillation or flutter. Am J Med. 2002;113:365-70.

4. Jacobs LG. Warfarin pharmacology, clinical management, and evaluation of hemorrhagic risk for the elderly. Cardiol Clin. 2008;26(2):157-67.

5. SAMSA GP, Matchar DB, Goldstein LB, Bonito AJ, Lux LJ, Witter DM, et al. Quality of anticoagulation management among patients with atrial fibrillation: Results of a review of medical records from 2 communities. JAMA: Internal Medicine, 2005; 160(7): 967-973.

6. Kalra L, Yu G, Perez I, Lakhani A, Donaldson N. Prospective cohort study to determine if trial efficacy of anticoagulation for strokeprevention in atrial fibrillation translates into clinical effectiveness.BMJ 2000;320:1236-1239.

7. Wallentin L, Yusuf S, Ezekowitz MD, Alings M, Flather M, Franzosi MG et al. Efficacy and safety of dabigatran compared with warfarin at different levels of international normalised ratio control for stroke prevention in atrial fibrillation: na analysis of the RELY trial. Lancet. 2010;376:975-83.

8. Glader EL, Sjölander M, Eriksson M, Lundberg M. Persistent use of secondary preventive drugs declines rapidly during the first 2 years after stroke. Stroke. 2010;41(2):397-401. 
9. Alexander J, Singh K.Inhibition of factor Xa: a potential target for the development of new anticoagulants. Am J Cardiovasc Drugs. 2005;5:279-290.

10. Sinnaeve PR, Werf FJV. Will oral antithrombin agents replace warfarin? Heart 2004; 90:827-828.

11.Fauci A, Braunwald E, Kasper D et al. Harrison's principles of internal medicine. New York: McGrawHill Medical. 2008.

12. Glund S, Moschetti V, Norris S, Stangier N, Schmohl $\mathrm{M}$, Ryn JV et al. A randomised study in healthy volunteers to investigate the safety, tolerability and pharmacokinetics of idarucizumab, a specific antidote to dabigatran. Thromb Haemost. 2015; 113: 943-51.

13. Connolly SJ, Crowther M, Eikelboom JW, Gibson M, Curnutte JT, Lawrence JH et al. Full study report of andexanet alfa for bleeding associated with Factor Xa inhibitors. N Engl J Med. 2019; 380:1326-1335.

14. Hart RG, Pearce LA, Aguilar MI. Meta-analysis: Antithrombotic Therapy to Prevent Stroke in Patients. Who Have Nonvalvular Atrial Fibrillation. Ann Intern Med. 2007;146:857-867.

15. Gage BF, Birman-Deych E, Kerzner R, Radford MJ, Nilasena DS, Rich MW. Incidence of intracranial hemorrhage in patients with atrial fibrillation who are prone to fall. Am J Med. 2005;118(6):612-7.

16. Karthikeyan G, Eikelboom JW. The CHADS2 score for stroke risk stratification in atrial fibrillation-friend or foe? Thromb Haemost. 2010;104(1):45-8.

17. Lip GY, Nieuwlaat R, Pisters R, Lane DA, Crijns HJ. Refining clinical risk stratification for predicting stroke and thromboembolism in atrial fibrillation using a novel risk factor-based approach: the euro heart survey on atrial fibrillation. Chest. 2010;137(2):263-72.

18. Fuster V, Ryden LE, Cannom DS, Crijns HJ, Curtis $\mathrm{AB}$, Ellenbogen KA et al. ACC/AHA/ESC 2006 Guidelines for the Management of Patients with Atrial Fibrillation: a report of the American College of Cardiology/American Heart Association Task Force on Practice Guidelines and the European Society of Cardiology Committee for Practice Guidelines (Writing Committee to Revise the 2001 Guidelines for the Management of Patients With Atrial Fibrillation): developed in collaboration with the European Heart Rhythm Association and the Heart Rhythm Society. Circulation. 2006;114(7):e257-354.

19. Pisters R, Lane DA, Nieuwlaat R, de Vos CB, Crijns HJ, Lip GY. A novel user-friendly score (HAS-BLED) to assess 1-year risk of major bleeding in patients with atrial fibrillation: the Euro Heart Survey. Chest. 2010;138(5):1093-100.

20. Connolly SJ, Ezekowitz MD, Yusuf S, Eikelboom J, Oldgren J, Parekh A, et al. Dabigatran versus Warfarin in Patients with Atrial Fibrillation. N Engl J Med. 2009;361:1139-51.
21. Patel MR, Mahaffey KW, Garg J, Pan G, Singer DE,Hacke W,et al. Rivaroxaban versus Warfarin in Nonvalvular Atrial Fibrillation. N Engl J Med. 2011;365:883-91.

22. Granger CB, Alexander JH, McMurray JJV, Lopes RD, Hylek EM, Hanna et al.Apixaban versus Warfarin in Patients with Atrial Fibrillation. N Engl J Med. 2011;365:981-92.

23. Giugliano RP, Ruff CT, Braunwald E, Murphy SA, Wiviott SD, Halperin JL et al. Edoxaban versus warfarin in patients with atrial fibrillation. $\mathrm{N}$ Engl $\mathrm{J}$ Med. 2013;369:2093-104.

24. Malik AH, Yandrapalli S, Aronow WS, Panza JA, Cooper HA. Meta-Analysis of Direct-Acting Oral Anticoagulants Compared With Warfarin in Patients $>75$ Years of Age. Am J Cardiol. 2019 Jun 15;123(12):2051-2057.

25. Kirchhof P, Benussi S, Kotecha D, Ahlsson A, Atar D, Casadei B et al. 2016 ESC Guidelines for the management of atrial fibrillation developed in collaboration with EACTS. Eur Heart J. 2016;37(38):2893-2962.

26. Lip GY, Windecker S, Huber K, KirchhofP, Marin F, Ten Berg JM etal. Management of antithrombotic therapy in atrial fibrillation patientspresenting with acute coronary syndrome and/or undergoingpercutaneous coronary or valve interventions: a joint consensusdocument of the European Society of Cardiology Working Groupon Thrombosis, European Heart Rhythm Association (EHRA), EuropeanAssociation of Percutaneous Cardiovascular Interventions(EAPCI) and European Association of Acute Cardiac Care (ACCA)endorsed by the Heart Rhythm Society (HRS) and AsiaPacificHeart Rhythm Society (APHRS). Eur Heart J. 2014;35(45):3155-79.

27. Gibson CM, Mehran R, Bode C, Halperin J, Verheugt FW, Wildgoose $\mathrm{P}$ et al. Prevention of Bleeding in Patients with Atrial Fibrillation Undergoing PCI. N Engl J Med. 2016;375(25):2423-34.

28. Cannon CP, Bhatt DL, Oldgren J, Lip GYH, Ellis SG,Kimura T, et al. Dual Antithrombotic Therapy with Dabigatran after PCI in Atrial Fibrillation. N Engl J Med 2017;377:1513-24.

29.Vranckx P, Valgimigli M, Eckardt L, Tijssen J, Lewalter T, Gargiulo Get al. Edoxaban-based versus vitamin $\mathrm{K}$ antagonist-based antithrombotic regimen after successful coronary stenting in patients with atrial fibrillation (ENTRUST-AF PCI): a randomised, open-label, phase 3b trial. Lancet. 2019 Oct 12;394(10206):1335-1343.

30. Lopes RD, Heizer G, Aronson R, Vora NA, Massaro T, Mehran R,et al. Antithrombotic Therapy after Acute Coronary Syndrome or PCI in Atrial Fibrillation. N Engl J Med. 2019 Apr 18;380(16):1509-1524. 
31. Yasuda S, Kaikita K, Akao M, Ako J, Matoba T, Nakamura $\mathrm{M}$ et al. Antithrombotic Therapy for Atrial Fibrillation with Stable Coronary Disease.N Engl J Med. 2019 Dec 19;381(25):2481.

32. Dunning J, Gao H, Chambers J, Moat M, Murphy G, Pagano D et al. Aortic valve surgery: marked increases in volume and significant decreases in mechanical valve use - an analysis of 41,227 patients over 5 years from the Society for Cardiothoracic Surgery in Great Britain and Ireland National database. J Thorac Cardiovasc Surg. 2011; 142:776-82.e3.

33. Nishimura RA, Otto CM, Bonow RO, Carabello BA, Erwin JP, Fleisher LA, et al. 2017 AHA/ACC Focused Update of the 2014 AHA/ACC Guideline for the management of patients with valvular heart disease: A report of the American College of Cardiology/American Heart Association Task Force on Clinical Practice Guidelines. Circulation 2017; 135(25):e1159-e1195.

34. Eikelboom JW, Connolly SJ, Brueckmann M, Granger $\mathrm{CB}$, Kappetein AP, Mack MJ et al. Dabigatran versus warfarin in patients with mechanical heart valves. N Engl J Med. 2013 Sep 26;369:1206-14.

35. Jamieson WR, von Lipinski O, Miyagishima RT, Burr LH, Janusz MT, Ling $\mathrm{H}$ et al. Performance of bioprostheses and mechanical prostheses assessed by composites of valve-related complications to 15 years after aortic valve replacement. J Thorac Cardiovasc Surg. 2006; 131:1267-73.

36. Serrano Junior CV, Fenelon G, Soeiro AM et al. Sociedade Brasileira de Cardiologia. Diretrizes Brasileiras de Antiagregantes Plaquetários e Anticoagulantes em Cardiologia. Arq Bras Cardiol. 2013;101 (3Supl.3): 1-93.

37. Carnicelli AP, De Caterina R, Halperin JL, Renda G, Ruff CT, Trevisan $\mathrm{M}$ et al. Edoxaban for the Prevention of Thromboembolism in Patients With Atrial Fibrillation and Bioprosthetic Valves. Circulation. 2017 Mar 28;135(13):1273-1275.

38.Guimarães PO, Pokorney SD, Lopes RD, Wojdyla DM, Gersh BJ, Giczewska A et al. Efficacy and safety of apixaban $v s$ warfarin in patients with atrial fibrillation and prior bioprosthetic valve replacement or valve repair: Insights from the ARISTOTLE trial. Clin Cardiol. 2019 May; 42(5):568-571.

39. Durães AR, Roriz OS, Nunes BA, Albuquerque FP, Bulhões FV, Fernandes AMS et al. Dabigatran versus warfarin after bioprosthesis valve replacement for the management of atrial fibrillation postoperatively: DAWA Pilot Study. Drugs R D. 2016 Jun;16(2):149-54.

40. Whitlock RP, Sun JC, Fremes SE, Rubens FD, Teoh KH. Antithrombotic and Thrombolytic Therapy for Valvular Disease: Antithrombotic Therapy and Prevention of Thrombosis, 9th ed: American College of Chest Physicians Evidence-Based Clinical Practice Guidelines. Chest 2012 Feb; 141(2): e576S-e600S.
41.Fox KA, Dabbous OH, Goldberg RJ, Pieper KS, Eagle KA, Van de Werf F et al. Prediction of risk of death and myocardial infarction in the six months after presentation with acute coronary syndrome: prospective multinational observational study (GRACE). BMJ. 2006;333(7578):1091.

42. Alexander JH, Lopes RD, James S, Kilaru R, He Y, Mohan $\mathrm{P}$ et al. Apixaban with antiplatelet therapy after acute coronary syndrome. N Engl J Med. 2011;365:699708.

43. Mega JL, Braunwald E, Wiviott SD, Bassand JP, Bhatt $\mathrm{DL}$, Bode $\mathrm{C}$ et al.Rivaroxaban in patients with a recent acute coronary syndrome. N Engl J Med. 2012 Jan 5;366(1):9-19.

44. Loh E, Sutton MS, Wun CC, Rouleau JL, Flaker GC, Gottlieb SS et al. Ventricular dysfunction and the risk of stroke after myocardial infarction. N Engl J Med. 1997;336(4):251-7.

45. Cleland JG, Findlay I, Jafri S, Sutton G, Falk R, Bulpitt $\mathrm{C}$ et al. The arfarin/Aspirin Study in Heart failure (WASH): a randomized trial comparing antithrombotic strategies in patients with heart failure. Am Heart J. 2004;148(1):157-64.

46. Massie BM, Collins JF, Ammon SE, Armstrong PW, Cleland JG, Ezekowitz $M$ et al; WATCH Trial Investigators. Randomized trial of warfarin, aspirin and clopidogrel in patients with chronic heart failure: the Warfarin and Antiplatelet Therapy in Heart Failure (WATCH) Trial. Circulation. 2009;119(12):1616-24.

47. Homma S, Thompson JL, Pullicino PM, Levin B, Freudenberger RS, Teerlink JR et al; WARCEF Investigators. Warfarin and aspirin in patients with heart failure and sinus rhythm. $N$ Engl J Med. 2012;366(20):1859-69.

48. You JJ, Singer DE, Howard PA, Lane DA, Eckman $\mathrm{MH}$, Fang $\mathrm{MC}$ et al. American College of Chest Physicians. Antithrombotic therapy for atrial fibrillation: Antithrombotic Therapy and Prevention of Thrombosis, 9th ed: American College of Chest Physicians Evidence-Based Clinical Practice Guidelines. Chest. 2012;141(2 Suppl):e531S-75S.

49. Andrade JP, Marin-Neto JA, Paola AA, Vilas-Boas F, Oliveira GM, Bacal F et al; Sociedade Brasileira de Cardiologia. I Diretriz latino americana para o diagnóstico e tratamento da cardiopatia chagásica. Arq Bras Cardiol. 2011;97(2 Suppl. 3):1-48.

50. Sousa AS, Xavier SS, Freitas GR, HasslocherMoreno A. Prevention strategies of cardioembolic ischemic stroke in Chagas' disease. Arq Bras Cardiol. 2008;91(5):306-10.

51. Rosa LV, Salemi VMC, Alexandre LM, Mady C. Miocardiopatia não compactada: uma visão atual. Arq. Bras. Cardiol., São Paulo, v. 97, n. 1, p. e13-e19, July 2011. 
52. Bocchi EA, Braga FGM, Ferreira SMA, Rohde LEP, Oliveira WA, Almeida DR, et al/Sociedade Brasileira de Cardiologia. III Diretriz brasileira de insuficiência cardíaca crônica. Arq Bras Cardiol. 2009; 93 (1 supl.1): $1-71$.

53. Rowin EJ, Hausvater A, Link MS, Abt P, Gionfriddo W, Wang $\mathrm{W}$ et al. Clinical Profile and Consequences of Atrial Fibrillation in Hypertrophic Cardiomyopathy. Circulation. 2017 Dec 19;136(25):2420-2436.

54. Camm AJ, Kirchhof P, Lip GY, Schotten U, Savelieva I, Ernst $\mathrm{S}$ et al. European Heart Rhythm Association;
European Association for Cardio-Thoracic Surgery. Guidelines for the management of atrial fibrillation: the Task Force for the Management of Atrial Fibrillation of the European Society of Cardiology (ESC). Eur Heart J. 2010;31(19):2369-429. Erratum in: Eur Heart J. 2011;32(9):1172.

55. Dangas GD, Tijssen JGP, Wöhrle J, Søndergaard L, Gilard M,Möllmann $\mathrm{H}$ et al. GALILEO Investigators. A Controlled Trial of Rivaroxaban after Transcatheter Aortic-Valve Replacement. N Engl J Med. 2020 Jan 9;382(2):120-129. 